\title{
fMRI identifies a network of structures correlated with retention of positive and negative emotional memory
}

\author{
TURHAN CANLI, ZUO ZHAO, JOHN E. DESMOND, GARY GLOVER, and JOHN D. E. GABRIELI \\ Stanford University, Stanford, California
}

\begin{abstract}
It is likely that the encoding of emotional experiences involves a complex network of interacting brain regions. The present study used fMRI to identify brain structures involved in the encoding of negative and positive emotional stimuli. Ten subjects viewed alternating blocks of emotionally negative and positive pictures and were tested for long-term recognition memory several months later. Recognition memory for negative and positive pictures was highly correlated. Brain reactivity to negative pictures was positively correlated with better recognition memory for both negative and positive pictures in the amygdala, the insula, and the right middle frontal gyrus. Brain reactivity to positive pictures was positively correlated with better recognition memory for both negative and positive pictures in the left anterior cingulate gyrus. Activation among these brain regions was highly correlated and suggests a network of structures that interact to encode either negative or positive emotional stimuli for longterm recognition memory. Two mechanisms by which this encoding may take place are discussed.
\end{abstract}

Salient emotional episodes are better remembered than nonemotional ones, presumably because of neural structures that enhance the encoding or retrieval of emotional experience. One brain structure believed to play a crucial role in the encoding of emotional experiences is the amygdala. An extensive literature on animals has documented the significance of the amygdala in the acquisition of conditioned fear and the enhancement of fearrelated reflexes, such as startle and eyeblink (Canli \& Brown, 1996; Davis, 1992; Davis, Rainnie, \& Cassell, 1994; LeDoux, 1995; Rogan, Staubli, \& LeDoux, 1997; Weisz, Harden, \& Xiang, 1992; Whalen \& Kapp, 1991), and studies of patients with amygdala damage have shown deficits in acquiring fear-conditioned responses (Bechara et al., 1995; LaBar, LeDoux, Spencer, \& Phelps, 1995). There is also a significant animal literature that suggests that emotional memory is modulated by the amygdala (Cahill \& McGaugh, 1998; McGaugh, Cahill, \& Roozendaal, 1996; Packard \& Teather, 1998), and patients with amygdala damage have failed to exhibit enhanced memory for emotional, as compared with neutral, segments of a story (Adolphs, Cahill, Schul, \& Babinsky, 1997; Cahill, Babinsky, Markowitsch, \& McGaugh, 1995). Functional imaging studies have shown that symptom provocation in patients with posttraumatic stress disorder activated the amygdala (Rauch et al., 1996), as did retrieval of emotional autobiographical memories (Fink et al., 1996). A positron emission tomography (PET) study demonstrated a significant correlation between

This research was supported by the NIH. G.G. is in the Department of Radiology at Stanford. Correspondence concerning this article should be addressed to T. Canli, Department of Psychology, Stanford University, Stanford, CA 94305 (e-mail: canli@psych.stanford.edu). amygdala activation at the time of encoding and subsequent free recall of emotionally arousing film clips 3 weeks later, with no such correlations for neutral film clips (Cahill et al., 1996). Another PET study (Hamann, Ely, Grafton, \& Kilts, 1999) has shown that amygdala activation during memory encoding is correlated not only with enhanced recognition memory for aversive visual scenes, but also with that for pleasant visual scenes (there was no correlation with neutral scenes).

Little is known about the role of any brain structures other than the amygdala in the encoding of emotional experiences. Several candidates for such a role include the anterior cingulate, the insula, the orbitofrontal cortex, and regions in the prefrontal lobe. These structures are known to respond to emotional stimuli (Canli, Desmond, Zhao, Glover, \& Gabrieli, 1998; Lane, Reiman, Ahern, Schwartz, \& Davidson, 1997; Phillips et al., 1997). Furthermore, activation in the anterior cingulate correlates with emotional awareness, which may be a significant determinant in memory encoding (Lane et al., 1998).

The present fMRI experiment was designed to assess the involvement of the amygdala, in concert with other structures, in the incidental encoding of emotional stintuli. Subjects viewed alternating blocks of negative and positive pictures that were similar in emotional arousal in the scanner and were tested for long-term recognition memory 2-14 months after encoding. We chose two emotional conditions of opposite valence (rather than one emotion and one neutral condition), in order to isolate brain regions whose activation is driven primarily by emotional valence, not by emotional stimuli in general. Correlation maps were constructed by mapping individual brains into a common template and correlating individual brain activations and recognition performances for neg- 
ative and positive pictures. We hypothesized that greater amygdala reactivity to negative pictures at encoding would correlate with recognition memory for these stimuli (Cahill et al., 1996; Hamann et al., 1999). The question of interest was whether any emotion-related areas other than the amygdala would be correlated with later recognition memory for either negative or positive emotional stimuli.

\section{METHOD}

\section{Subjects}

Fourteen right-handed healthy female volunteers (mean age, 25.6 years; range, 19-42 years) participated in the encoding phase of this study. The data from these subjects were previously reported with respect to the role of emotional arousal on brain activation patterns during encoding (Canli et al., 1998). Of these, 10 subjects were available at the time of memory testing (mean age, 27.7 years; range, $21-42$ years). All the correlations and correlation maps reported here are based on these 10 subjects.

\section{Encoding Procedure}

The subjects passively viewed 20 negative and 20 positive pictures selected from a standardized set of pictures (IAPS; Lang \& Greenwald, 1993) and were unaware that they were participating in a memory experiment. Negative pictures included images of angry or crying people, spiders, guns, and a cemetery. Positive pictures included images of a happy couple, puppies, foods like ice cream and brownies, and urban sunsets. Images were presented in five alternating blocks of 4 pictures each. The order of positive and negative blocks was alternated across subjects. Each picture was presented for $7,500 \mathrm{msec}$, with an interstimulus interval of $1,125 \mathrm{msec}$. Total scan time was $345 \mathrm{sec}$.

\section{Emotion ratings}

As a manipulation check, the subjects were shown the same sequence of stimuli immediately after the scan and were asked to rate each picture on a visual scale (Lang \& Greenwald, 1993) with regard to valence and arousal.

\section{Recognition Test Procedure}

The subjects were invited back to the laboratory between 2 and 14 months after the encoding session to participate in a recognition test. The wide range of delay periods between encoding and recognition testing was due to the fact that some subjects were unavailable for memory tests for extended periods (e.g., because of study abroad or a move after graduation). In this recognition test, a total of 80 pictures were shown: the original set of 40 negative and positive pictures seen during encoding, plus an additional set of 20 negative and 20 positive novel pictures. Each picture was displayed for up to $7,500 \mathrm{msec}$, and the order of the pictures was randomized. The subjects were asked whether they recognized each picture as being one they had seen previously while in the scanner.

\section{MRI}

Data were acquired in a $1.5 \mathrm{~T}$ GE Signa MR imager, which was used to measure blood-oxygen level-dependent (BOLD) contrast (Ogawa, Lee, Nayak, \& Glynn, 1990). For structural images, 16 coronal slices of 3-mm thickness were imaged, using a T1-weighted flow-compensated spin-echo sequence $(\mathrm{TR}=500 \mathrm{msec}$, minimum $\mathrm{TE}$ ). Functional images were obtained using a $\mathrm{T} 2{ }^{*}$-sensitive threedimensional gradient echo spiral sequence with four interleaves $\left(\mathrm{TR}=90 \mathrm{msec}, \mathrm{TE}=40 \mathrm{msec}\right.$, flip angle $=22^{\circ}, \mathrm{FOV}=36 \mathrm{~cm}$, inplane resolution $=2.35 \mathrm{~mm}^{2}$, acquisition time $=4.32 \mathrm{sec}$ per frame, number of frames $=80 ;$ Glover $\&$ Song, 1998). A whole-head coil was used for all the subjects. Head movement was minimized by using a bite-bar with each subject's dental impression. A standard algorithm for motion correction (Woods, Cherry, \& Mazziotta, 1992) was used. Functional activation ( $z$ score) maps were based on a correlation between the signal intensity of each pixel and a reference function that was computed by convolving a square-wave at the task frequency (alternating blocks of negative and positive pictures) with a data-derived estimate of the hemodynamic response function (Friston, Jezzard, \& Turner, 1994).

\section{Correlation Maps}

For each of the 10 subjects in this study, the subject's original $z$ score map for each slice was transformed into a common coordinate system by morphing the data onto a common template. Then, for each pixel in the template, a correlation was computed between subjects' $z$ scores and performance measures (emotion ratings of valence and arousal and memory scores). Thus, each pixel was represented by two columns: (1) positive or negative $z$ scores, representing, for each subject, the degree of correlation between activation of that pixel in phase (positive pictures) or out of phase (negative pictures), respectively, and (2) performance scores, representing individual differences in emotional experience or memory performance. To control for Type I errors, we performed a cluster analysis (Xiong, Gao, Lancaster, \& Fox, 1995), with a conjoint intensity and spatial extent threshold that yielded a $p<.05$ (twotailed) significance level over the entire image. Significant clusters were color coded and displayed by projection onto an averaged T1weighted anatomical image of each slice.

\section{RESULTS}

\section{Emotion Ratings}

Figure 1 shows that the subjects experienced positive and negative picture sets as being different in valence and in arousal. Rating emotional valence on a scale from 1 (unhappy) to 9 (happy), positive pictures were rated as $6.69 \pm 0.75$ (mean $\pm S D$ ), and negative pictures were rated as $3.42 \pm 0.48$. These ratings were significantly different from each other in a paired $t$ test $(t=9.91, p<$ $.0001)$. Rating emotional arousal on a scale from $1(\mathrm{calm})$ to 9 (aroused), positive pictures were rated as $4.13 \pm 1.51$, and negative pictures were rated as $5.14 \pm 1.45$. These ratings were significantly different from each other in a paired $t$ test $(t=2.91, p<.02)$.

\section{Memory Performance}

Recognition memory was calculated by subtracting the percentage of false alarms from the percentage of hits, so that chance performance would be represented by a score of 0 and perfect memory by a score of 100 . A paired $t$ test showed no significant difference in recognition memory for negative (mean $=40.1, S D=20.39$ ) and positive (mean $=37.5, S D=22.88$ ) pictures $[t(9)=.77, p=$ .46]. Indeed, calculation of correlation coefficients and Fisher's $r$-to- $z$ conversions showed that recognition memory for negative items was highly correlated with recognition memory for positive items across subjects $(r=$ $.88, p<.0002$ ), so that subjects who remembered negative pictures well also remembered positive pictures well. 


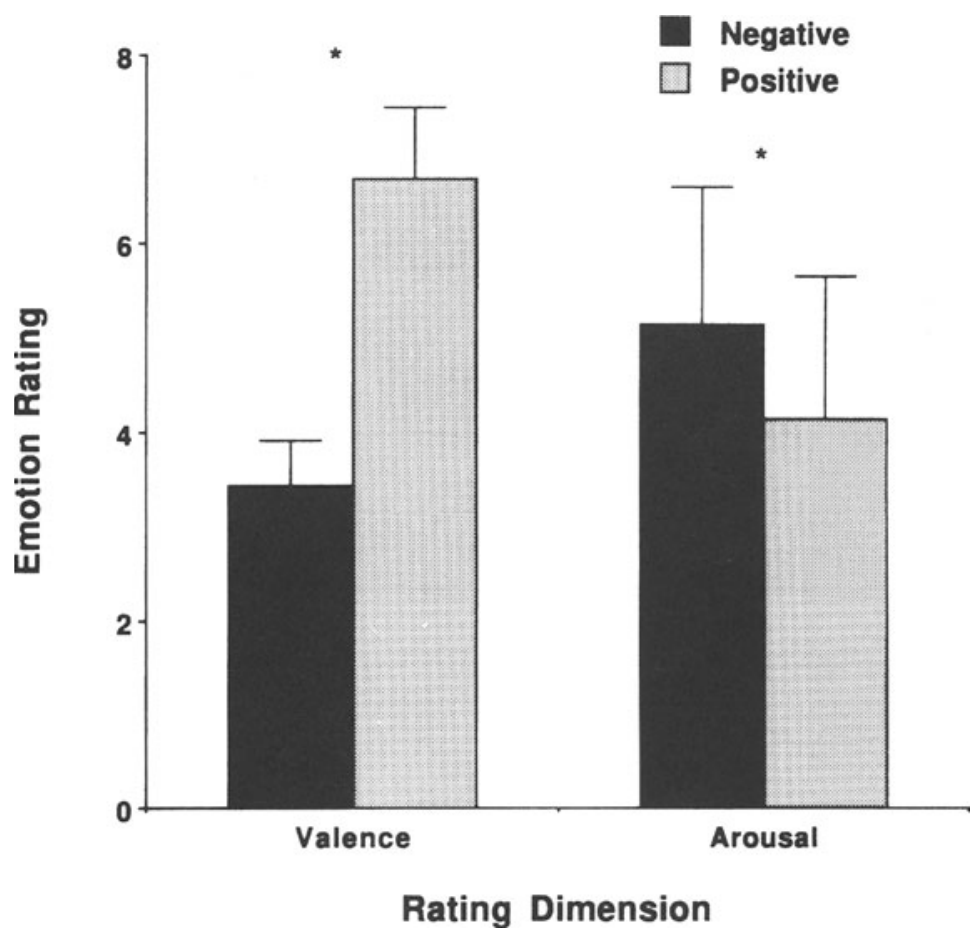

Figure 1. Mean subject ratings of emotional valence and arousal for negative and positive pictures. Each subject rated each picture individually for emotional valence and arousal. For each subject, a mean rating of emotional valence and arousal was calculated. Paired $t$ tests were conducted to assess whether mean ratings for negative and positive pictures were significantly different for valence or arousal.

\section{Memory Performance as a Function of Retention Interval}

The mean retention interval between encoding and recognition test sessions was 211 days (range, 67-400 days; $\mathrm{SD}, 135$ days). To assess whether memory performance was significantly dependent on the duration of the retention interval, correlation coefficients and Fisher's $r$-to- $z$ conversions were calculated between retention interval and each of six memory measures (hits, false alarms, and recognition scores for negative and positive pictures). There was no significant correlation between retention interval and any of the memory measures (range for $r$, -.36 to .28 ; range for $p, .32$ to .87 ).

\section{Memory Performance as a Function of Valence and Arousal Ratings}

To assess whether memory performance was significantly correlated with subjects' ratings for valence and arousal, correlation coefficients and Fisher's $r$-to- $z$ conversions were calculated between subjects' ratings (valence and arousal) and memory measures (hits, false alarms, and recognition scores) for negative and positive pictures, respectively. There was no significant correlation between the valence and arousal ratings for negative pictures and any memory measure, although there was a near-significant trend toward a higher number of hits with lower (i.e., more negative) valence ratings ( $r=-.61$, $p=.06)$. There was no significant correlation between the valence and arousal ratings for positive pictures and any memory measure. Thus, the subjective experience of emotional valence or arousal did not predict subsequent memory performance for either positive or negative pictures.

\section{Correlation of Recognition Memory and Brain Reactivity to Valenced Stimuli}

Table 1 lists clusters where brain reactivity to negative and/or positive pictures correlated significantly with subsequent recognition memory for these stimuli. Areas where greater reactivity to negative (relative to positive) pictures correlated with recognition memory included the right middle frontal gyrus and the left inferior frontal gyrus, as well as (bilaterally) the insula and the amygdala. The only area where greater reactivity to positive (relative to negative) pictures correlated with recognition memory was the anterior cingulate. For all of these regions, brain activation correlated strongly with recognition memory for both negative and positive pictures (negative $r$ values ranged from -.65 to -.94 ; positive $r$ values ranged from .95 to .96), although not all of these regions passed the clustering threshold, which required both significant 


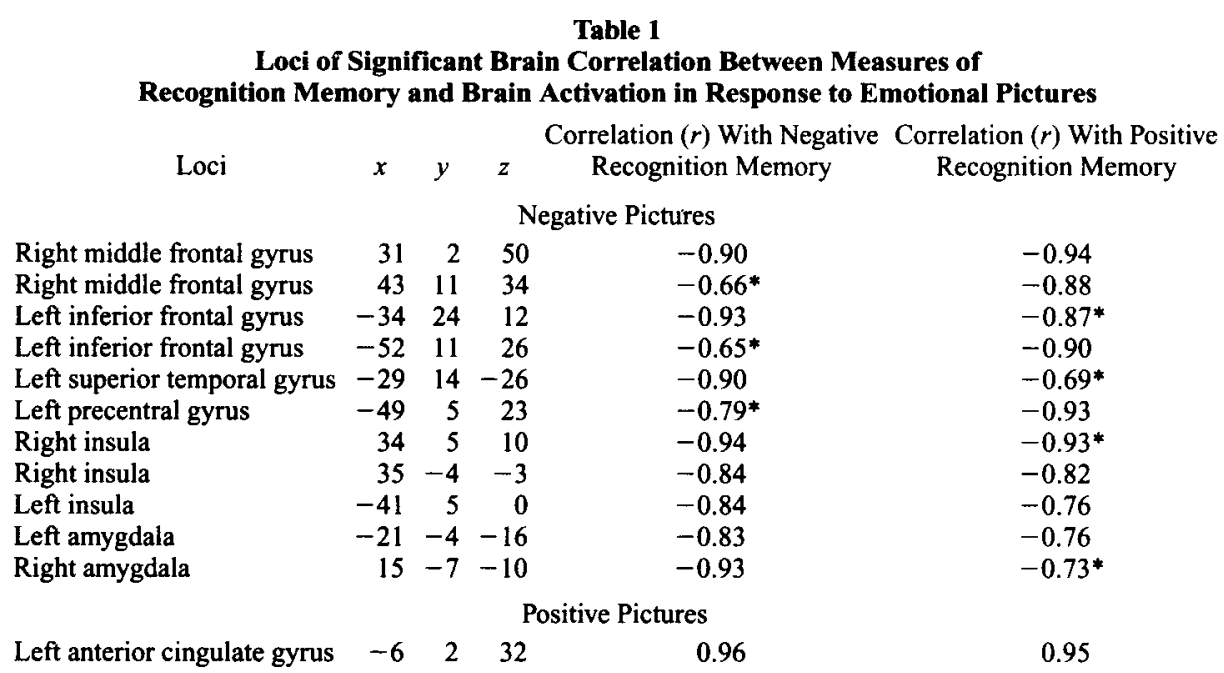

Note-Three-dimensional clusters of significant correlations were determined by the methods of Xiong, Gao, Lancaster, and Fox (1995). Talairach coordinates of these clusters were determined by the methods of Desmond and Lim (1997). Regions are identified by name of location and coordinates in the brain atlas of Talairach and Tournoux (1988). $x$, distance in millimeters to the right $(+)$ or left $(-)$ of midline; $y$, distance anterior $(+)$ or posterior $(-)$ to the anterior commissure; $z$, distance superior $(+)$ or inferior $(-)$ to a horizontal plane through the anterior and posterior commissures. *Denotes a correlation that did not pass significance threshold (in correlational strength or size of spatial extent) in the cluster analysis.

correlational strength and a significant number of neighboring pixels exhibiting such a correlation. Figure 2 shows several regions where brain reactivity correlated significantly with recognition memory for both positive and negative pictures and where clusters exhibiting such a correlation passed the clustering threshold.

Figures 3 and 4 show scatterplots from clusters in Figure 2 and from control locations, respectively. Control regions were similar in size to significant clusters and were chosen to control for laterality (e.g., the left vs. the right middle frontal gyrus), regional specificity (e.g., the superior frontal versus the middle frontal gyrus), and neural activation (e.g., white matter vs. gray matter). Scatterplots illustrate the relationship between brain reactivity to emotional pictures and negative (left panel) or positive (right panel) recognition memory. These scatterplots show that the left amygdala, the right insula, and the middle frontal gyrus, but not control locations, exhibited a greater reactivity to negative (relative to positive) pictures that correlated significantly with recognition memory. Only the anterior cingulate exhibited greater reactivity to positive (relative to negative) pictures that correlated significantly with recognition memory.

To determine whether separate clusters were interrelated in their activation to emotional pictures, correlations were calculated between all the regions that showed a significant correlation with both negative and positive recognition memory. Table 2 illustrates that the left amygdala, the right insula, and the right middle frontal gyrus all respond similarly to stimuli of the same valence. Thus, in subjects who exhibited greater amygdala reactivity to negative stimuli, the insula and the right middle frontal gyrus also exhibited greater reactivity to negative stimuli. The table also illustrates that activation in the left anterior cingulate responded to valenced stimuli in a direction that was opposite that for the amygdala, the insula, and the middle frontal gyrus. Thus, in subjects who exhibited greater amygdala reactivity to negative (relative to positive) stimuli, the anterior cingulate exhibited greater reactivity to positive (relative to negative) stimuli.

\section{DISCUSSION AND CONCLUSION}

We found several brain structures that are involved in the encoding of negative and positive emotional experiences into long-term memory. Greater reactivity to negative (relative to positive) pictures correlated with better recognition memory in a number of structures, including the right middle and the left inferior frontal gyrus and (bilaterally) the amygdala and the insula. Greater reactivity to positive (relative to negative) pictures correlated with better recognition memory in the left anterior cingulate gyrus. Recognition memory for both negative and positive pictures was highly correlated across subjects, so that individuals with high recognition memory for negative pictures also exhibited high recognition memory for positive pictures. As a consequence, several brain structures (the left amygdala, the left and the right insula, the right middle frontal gyrus, and the left anterior cingulate) exhibited strong and significant (i.e., exceeding clustering threshold) correlations with recognition memory for both negative and positive pictures.

Among the structures found to encode emotional memory, the amygdala has received the most attention to 


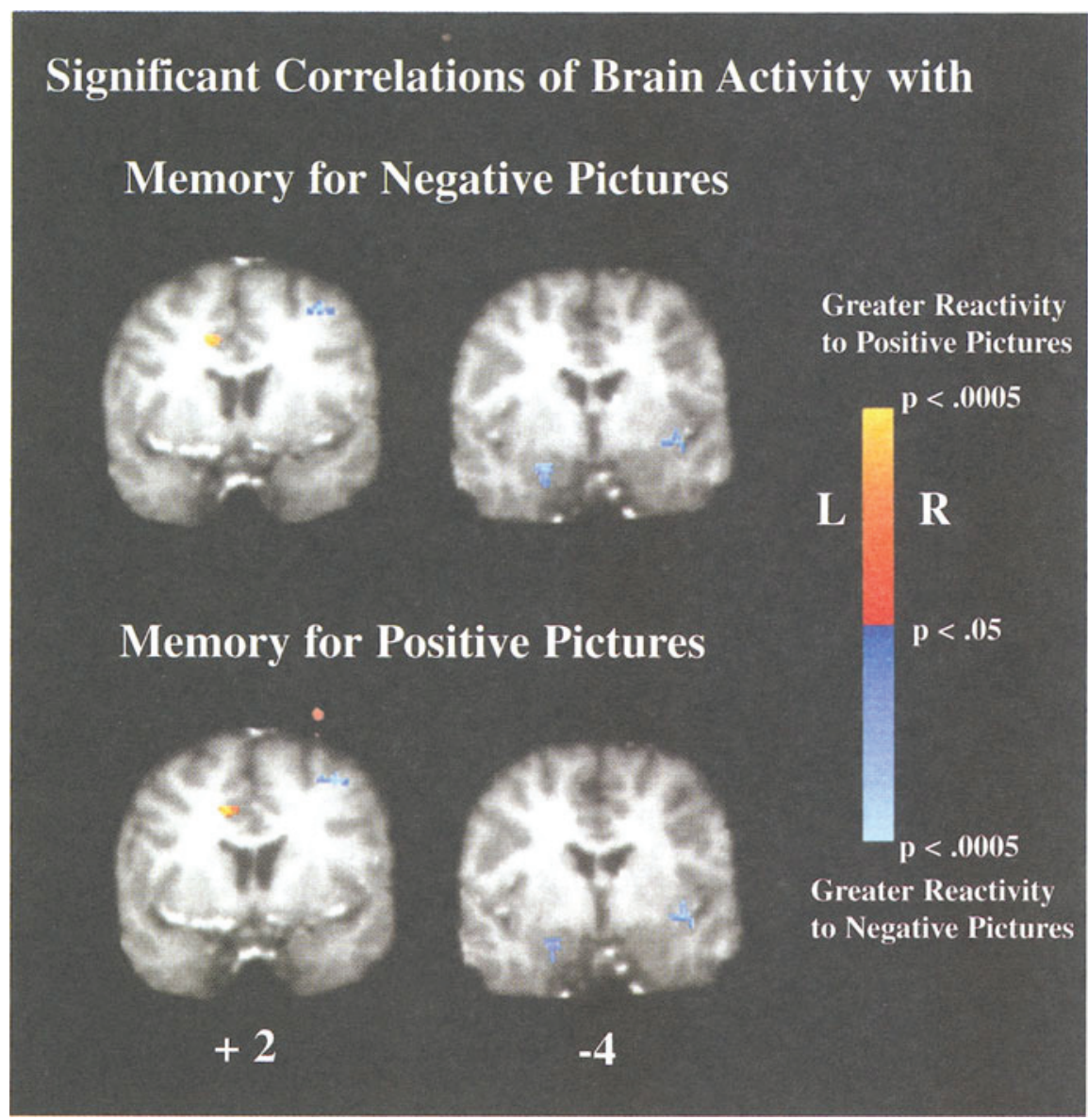

Figure 2. Loci of significant correlations between brain activation to emotional pictures and recognition memory. Shown are coronal slices illustrating loci of significant $(p<.05)$ correlations between brain activation to emotional pictures and recognition memory for negative pictures (top row) or positive pictures (bottom row). Loci colored in yellow represent regions of significant correlations between recognition memory and reactivity to positive (relative to negative) pictures. Loci colored in blue represent regions of significant correlations between recognition memory and reactivity to negative (relative to positive) pictures.

date. A considerable amount of literature on animals has demonstrated the association between amygdala functioning and emotional memory in a number of paradigms, including fear-potentiated startle (Davis, 1992), freezing (LeDoux, 1995), avoidance learning (Cahill \& McGaugh, 1990; Nagel \& Kemble, 1976), and eyeblink facilitation (Canli \& Brown, 1996; Weisz et al., 1992; Whalen \& Kapp, 1991). Human patient studies have associated amygdala damage with deficits in fear conditioning (Bechara et al., 1995; LaBar et al., 1995). There is also a large literature on animals pointing to the modulatory role of the amygdala in enhancing emotional memory (Cahill \& McGaugh, 1998; McGaugh, Cahill, \& Roozendaal, 1996; Packard \& Teather, 1998). Patient studies have reported a failure to show enhanced memory for emotional aspects of a story (Adolphs et al., 1997; Cahill et al., 1995).
Our data agree in large part with two previous neuroimaging (PET) studies that found significant correlations between amygdala activation and subsequent memory performance. One PET study (Cahill et al., 1996) reported a significant correlation between amygdala reactivity to emotionally arousing negative, but not neutral, films and free-recall memory 3 weeks later. Another PET study (Hamann et al., 1999) found significant correlations between amygdala activity during encoding of negative and positive, but not interesting or neutral, visual stimuli and subsequent recognition memory 4 weeks later. Thus, both PET and fMRI demonstrate a role for the amygdala in the encoding of emotional memory. This role applies to the encoding of emotionally arousing still and moving visual stimuli, to retention intervals from 3 weeks to 14 months, and to free recall and recognition memory. 


\section{Significant Clusters}
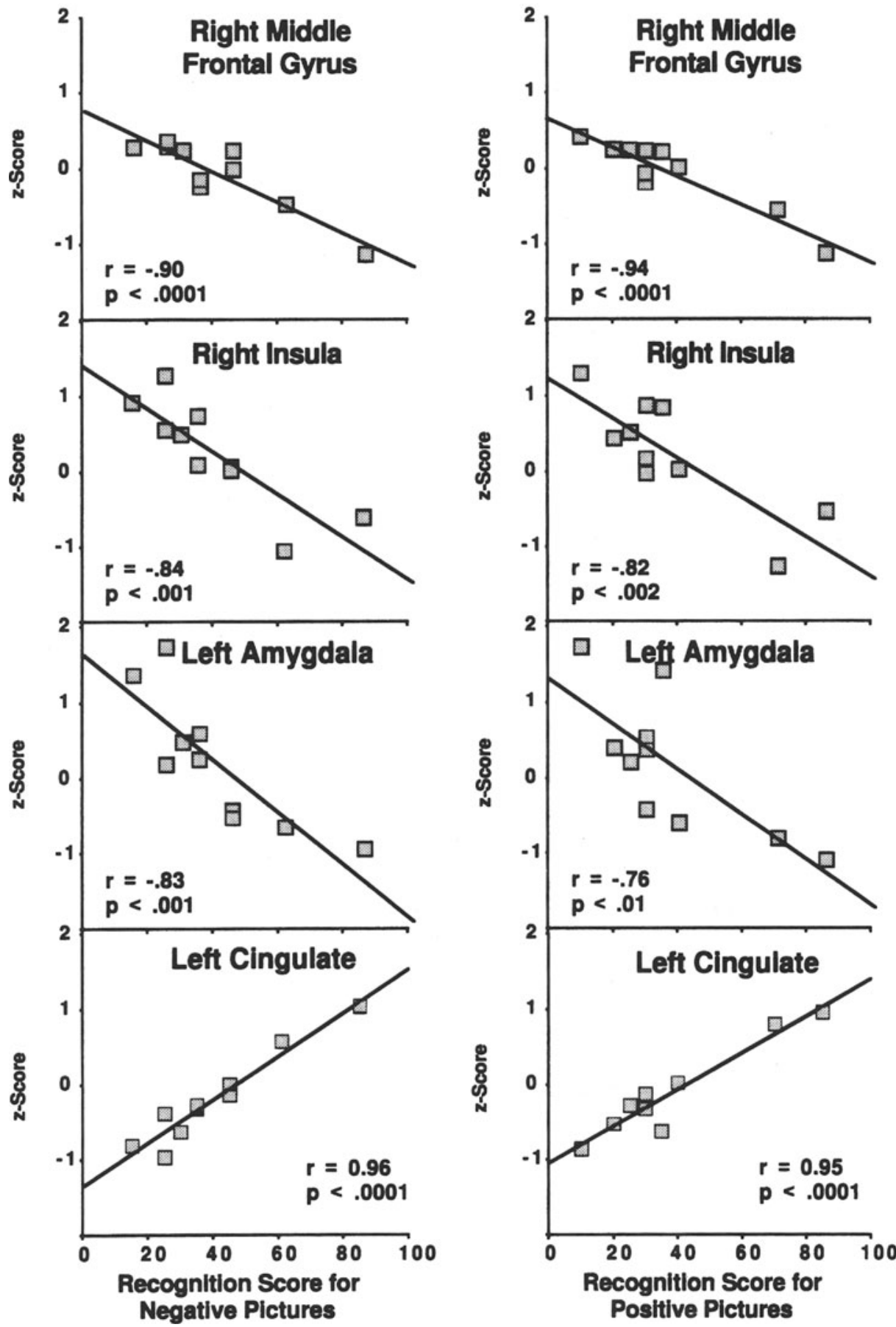

Figure 3. Scatterplots of clusters with significant correlations between recognition memory and brain activation to emotional pictures. Shown are scatterplots from clusters of significant correlations between subjects' recognition memory for negative pictures (left column) or positive pictures (right column) and brain activation to emotional pictures. Positive $z$ scores represent greater activation to positive, relative to negative, pictures. Negative $z$ scores represent greater activation to negative, relative to positive, pictures. Positive correlations indicate better recognition memory with greater activation to positive (relative to negative) pictures. Negative correlations indicate better recognition memory with greater activation to negative (relative to positive) pictures. 


\section{Control Locations}
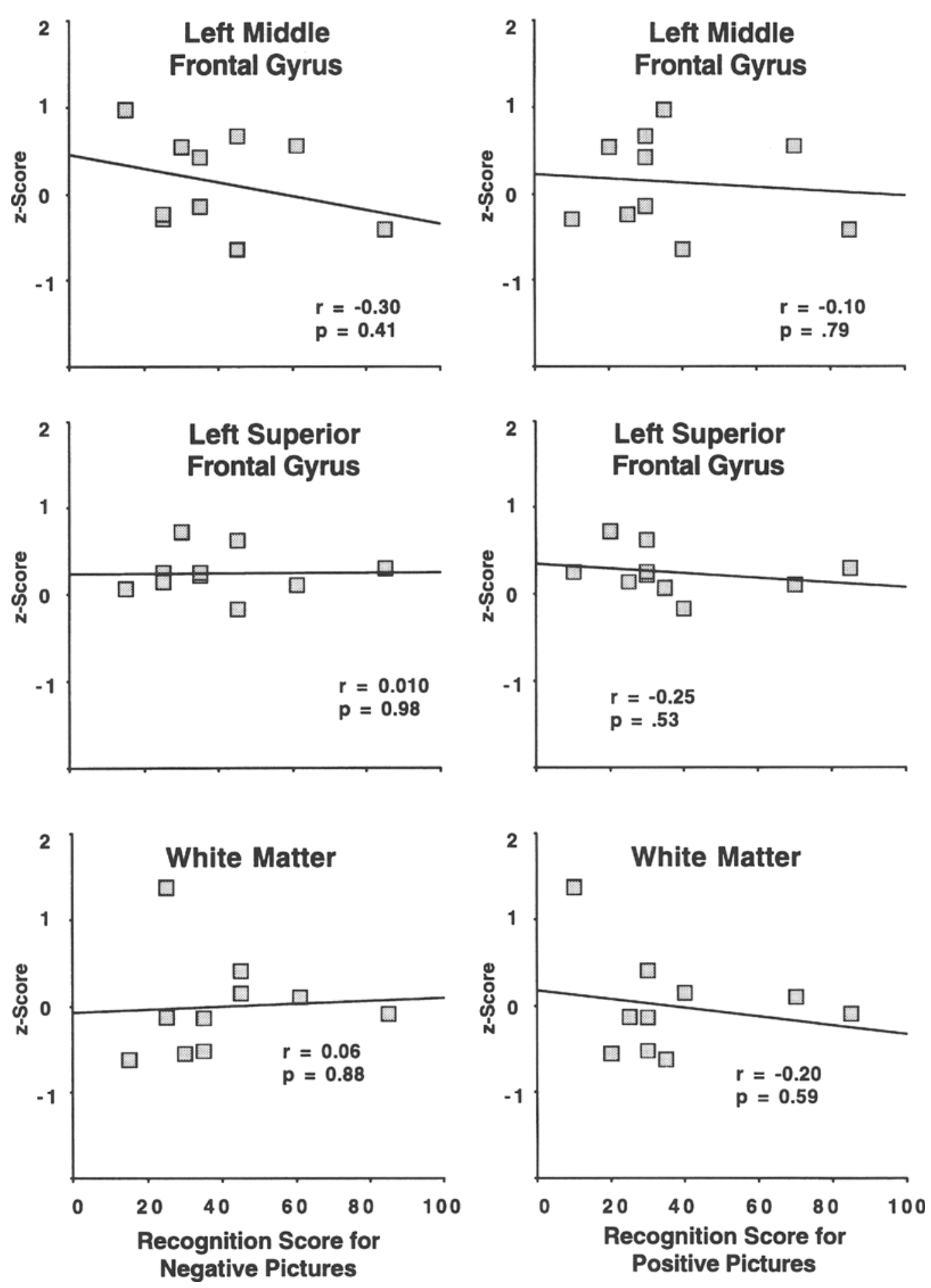

Figure 4. Scatterplots of control locations illustrating correlations between recognition memory and brain activation to emotional pictures. Shown are scatterplots from control locations. No significant correlations were found in these locations between subjects' recognition memory for negative pictures (left column) or positive pictures (right column) and brain activation to emotional pictures. Positive $\boldsymbol{z}$ scores represent greater activation to positive, relative to negative, pictures. Negative $z$ scores represent greater activation to negative, relative to positive, pictures. Positive correlations indicate better recognition memory with greater activation to positive (relative to negative) pictures. Negative correlations indicate better recognition memory with greater activation to negative (relative to positive) pictures. 
Table 2

Correlation Matrix: Clusters of Significant Correlation Between

Brain Reactivity and Recognition Memory for Emotional Pictures

Control Area: Control Area:

Right Middle Left Middle Left Superior Control Area: Left Cingulate Amygdala Right Insula Frontal Gyrus Frontal Gyrus Frontal Gyrus White Matter

\author{
Left cingulate \\ Left amygdala \\ Right insula \\ Right middle frontal gyrus \\ Control area: Left middle frontal gyrus \\ Control area: Left superior frontal gyrus \\ Control area: White matter
}

Left cingulate

Left amygdala

Right insula

Right middle frontal gyrus

Control area: Left middle frontal gyrus

Control area: Left superior frontal gyrus

Control area: White matter

\begin{tabular}{|c|c|c|c|c|c|c|}
\hline \multirow{3}{*}{$\mathrm{x}$} & \multicolumn{2}{|c|}{ Negative Pictures } & \multirow[b]{2}{*}{-0.87} & \multirow[b]{2}{*}{-0.19} & \multirow[b]{2}{*}{-0.05} & \multirow[b]{2}{*}{-0.09} \\
\hline & -0.89 & -0.91 & & & & \\
\hline & $\mathrm{x}$ & 0.86 & 0.64 & 0.24 & 0.04 & 0.17 \\
\hline & & $\mathrm{x}$ & 0.72 & -0.02 & 0.08 & 0.11 \\
\hline & & & $\mathbf{x}$ & 0.29 & 0.13 & 0.14 \\
\hline & & & & $\mathrm{x}$ & 0.41 & -0.34 \\
\hline & & & & & $\mathrm{x}$ & -0.02 \\
\hline & & & & & & \\
\hline $\mathrm{x}$ & -0.89 & -0.91 & -0.91 & -0.18 & -0.16 & -0.1 \\
\hline & $\mathrm{x}$ & 0.86 & 0.7 & 0.24 & 0.03 & 0.15 \\
\hline & & $\mathrm{x}$ & 0.71 & -0.09 & 0.06 & 0.1 \\
\hline & & & $x$ & 0.24 & 0.15 & 0.21 \\
\hline & & & & $\mathrm{x}$ & 0.41 & -0.34 \\
\hline & & & & & $\mathrm{x}$ & -0.02 \\
\hline
\end{tabular}

Note-The upper part (for negative pictures) is based on $z$ scores from activation clusters that were found to correlate significantly with negative recognition memory, and the lower part (for positive pictures) is based on $z$ scores from activation clusters that were found to correlate significantly with positive recognition memory. Positive correlations indicate that two brain regions exhibited greater reactivity toward stimuli of the same valence. Negative correlations indicate that two brain regions exhibited greater reactivity toward stimuli of the opposite valence.

The study by Cahill et al. (1996), consistent with the data presented here, reports that, despite a significant correlation between amygdala activation and recognition memory, amygdala activation (as measured in $z$ scores) during encoding was not significantly greater for negative than for neutral pictures. This finding is also consistent with several other studies that failed to detect amygdala activation in response to emotional stimuli. For instance, Fischer and colleagues (Fischer, Wik, \& Fredrikson, 1996) found no amygdala activation when they scanned subjects who reexperienced a bank robbery by means of watching a surveillance video. Two PET studies failed to detect amygdala activation in a fear-conditioning paradigm (Fredrikson, Wik, Fischer, \& Andersson, 1995; Hugdahl et al., 1995), contradicting data from animal studies (Davis, 1992; LeDoux, 1995) and from studies of human patients with amygdala lesions (Bechara et al., 1995; LaBar et al., 1995; Phelps \& Anderson, 1997). A number of PET studies also failed to detect amygdala activation in response to phobic stimulation (Fredrikson, Wik, Annas, Ericson, \& Stone-Elander, 1995; Fredrikson et al., 1993; Rauch et al., 1995; Wik et al., 1993). These studies suggest that the amygdala may not be necessary for the experience of negative emotions.

On the other hand, Hamann et al. (1999) found significantly greater amygdala activation in responses to emotional than to neutral pictures. Other studies have reported amygdala activation during the retrieval of posttraumatic memories in humans (Rauch et al., 1996) and emotional autobiographical memories (Fink et al., 1996), the viewing of unpleasant pictures or films (Irwin et al., 1996;
Lane, Reiman, Bradley et al., 1997; Reiman et al., 1997), the experience of the mood of sadness (Schneider et al., 1995), procaine-induced intense fear (Ketter et al., 1996), and depression (Drevets et al., 1992). Thus, the role of the amygdala in the experience of emotions remains to be elucidated.

Consistent with Hamann and colleagues (Hamann et al., 1999), we found that activation in both the left and the right amygdala correlated significantly with subsequent memory for emotional stimuli. Involvement of the left amygdala in the encoding of emotional memory is consistent with other studies that have reported left amygdala activation during the experience of sadness and happiness (Schneider et al., 1997; Schneider et al., 1995), intense fear (Ketter et al., 1996), and depression (Drevets et al., 1992) or in response to negative visual stimuli (Taylor et al., 1998) and fearful faces (Breiter et al., 1996; Morris et al., 1996). Involvement of the right amygdala in the encoding of emotional memory is consistent with Cahill et al. (1996), who reported a significant correlation between right amygdala activation and emotional memory. The difference between bilateral amygdala correlations (Hamann et al., 1999; the present study) and right amygdala correlations (Cahill et al., 1996) may be due to the choice of stimuli (still pictures vs. film clips) or performance measure (recognition memory vs. free recall). Future work needs to identify the determinants of laterality patterns in the amygdala during encoding of emotional stimuli.

This study addressed whether a network of structures is involved in the encoding of emotional memory. In- 
deed, we found a set of interconnected limbic and cortical structures, including the insula and the cingulate cortex, that showed significant correlations between activation and emotional memory and among each other. Activation of the insula has been observed in response to facial expressions of disgust (Phillips et al., 1997) but has not previously been linked to emotional memory. Activation of the cingulate, on the other hand, has been correlated with memory for pleasant stimuli (Hamann et al., 1999), perhaps because of its involvement in emotional awareness (Lane et al., 1998). We did not find significant correlations between activity in the orbitofrontal cortex and emotional memory, such as were previously noted by Cahill and colleagues (Cahill et al., 1996). This may reflect poor signal-to-noise conditions, because fMRI signals are difficult to obtain from the orbitofrontal cortex.

Of those loci where brain activation to emotional pictures correlated with subsequent recognition memory, most showed greater reactivity to negative (relative to positive) pictures (e.g., the amygdala, the insula, and the middle frontal gyrus), whereas only the anterior cingulate showed greater reactivity to positive (relative to negative) pictures. Because both conditions contained emotional stimuli that only differed in valence, it is likely that activation patterns that emerge in one condition would identify structures that participate in the processing of emotional valence, not of emotion in general. This is because structures that participate in the processing of emotion regardless of valence would be equally active in both conditions and, therefore, would not significantly correlate with either condition. Although the advantage of this design is that it isolates activations specific to emotional valence, the disadvantage of this design is that it lacks a neutral baseline condition. The lack of a neutral baseline condition complicates the interpretation of the data, because an increase in activation to negative stimuli is equivalent to a decrease in activation to positive stimuli. Future work should include both negative and positive emotional conditions, as well as a neutral baseline condition.

In the absence of a neutral baseline, we offer two interpretations for the activation pattern observed in this study. One interpretation is that there are two separate systems encoding negative and positive pictures. In one system, the level of activation in some structures (e.g., the amygdala, the insula, and the middle frontal gyrus) in response to negative (relative to positive) pictures determines subsequent recognition memory for negative pictures. In another system, the level of activation in the anterior cingulate in response to positive (relative to negative) pictures determines subsequent recognition memory for positive pictures. If there are two systems, patients with Urbach-Wiethe disease that produced selective amygdala damage should show impaired memory for negative, but not for positive, stimuli, whereas patients with damage to the anterior cingulate should show the opposite pattern.

The alternative interpretation is that there is a single system that encodes negative pictures, in which some components (the amygdala, the insula, and the middle frontal gyrus) inhibit another component (the anterior cingulate). This interpretation is derived from the fact that, in a blocked design, where brain activation to one set of stimuli is quantified relative to brain activation to another set of stimuli, an increase in activation to positive stimuli in the anterior cingulate is equivalent to a decrease in activation to negative stimuli. ${ }^{1}$ According to this interpretation, greater reactivity during exposure to negative, but not to positive, pictures is the determinant for subsequent recognition memory. The question, however, is how activation in response to negative pictures could enhance memory for positive pictures. One possibility is that the activations that occur during the presentation of negative pictures apply to the entire study episode. Because negative and positive pictures were seen in intertwined blocks of $30 \mathrm{sec}$ per block, it is possible that the memory process that was activated during presentation of negative pictures may have stayed engaged long enough to affect memory encoding for positive pictures, as well. This effect would not be seen in an experimental design where negative and neutral films were seen in different episodes separated by 3-7 days (as in Cahill et al., 1996).

This alternative interpretation implies that the correlation between cingulate activation and subsequent recognition memory is driven by exposure to negative, not positive, stimuli. Therefore, a study in which subjects encode only positive stimuli should fail to find a correlation between cingulate activation and subsequent memory. The data by Hamann et al. (1999), however, speak against this prediction. To the extent that the amygdala plays a principal role in the activation of memory processes encoding both negative and positive stimuli, we further predict that patients with selective amygdala damage owing to Urbach-Wiethe disease should exhibit impairments in memory for both negative and positive stimuli in a blocked design. To date, we are not aware of any data that would comment on this prediction.

Unlike Hamann et al. (1999), we did not find a significant correlation between amygdala activation to positive emotional stimuli and memory for these stimuli. This may be due to the design of our experiment, in which amygdala activation to negative and to positive stimuli were directly compared against each other. If similar regions within the amygdala respond to negative and positive stimuli, a direct comparison would fail to reveal significant activation to positive stimuli. Indeed, this explanation is supported by data from Hamann et al., who found that areas of amygdala activation elicited by positive pictures do overlap with areas activated by negative pictures and that direct contrasts (positive minus negative and negative minus positive) do not yield differences in the area of the amygdala (S. B. Hammann, June 15, 1999, personal communication).

Although our data show that amygdala activation correlates with subsequent recognition memory for emotional stimuli, they do not identify the location where emotional memory is stored. This issue remains the topic 
of considerable controversy (McGaugh et al., 1996; Muller, Corodimas, Fridel, \& LeDoux, 1997; Rogan et al., 1997) and will probably not be resolved until both encoding and retrieval tasks are employed within one study.

Similarly, our data do not address whether or how amygdala activation affects other structures identified in this study. Evidence from animal studies suggests that the amygdala modulates memory storage processes in other brain regions, such as the hippocampus and the caudate nucleus (Ikegaya, Saito, \& Abe, 1996; Packard, Cahill, \& McGaugh, 1994; Packard \& Teather, 1998), two structures that support different forms of learning (Packard \& McGaugh, 1996). Hamann et al. (1999) found correlations that were consistent with the view that the amygdala modulates episodic memory for emotional stimuli via the hippocampus. Our study cannot speak to this issue, because we did not collect data from hippocampal regions. Future work will use single-trial (event-related) fMRI to investigate the relationship between the amygdala and the hippocampus in greater detail.

Future work also needs to assess the role of gender in the encoding of emotional experiences. Women are more likely to report intense emotional experiences (Shields, 1991) and to show more physiological reactivity in concordance with valence judgments than do men (Lang, Greenwald, Bradley, \& Hamm, 1993). PET imaging studies find gender differences even at rest (Gur et al., 1995) and differential activation patterns in emotion-related tasks. In one study (Pardo, Pardo, \& Raichle, 1993), men and women were asked to imagine or recall a situation that would make them sad: Women showed bilateral inferior and orbitofrontal activation, whereas men showed left-lateralized activation in the same regions. In another study (George, Ketter, Parekh, Herscovitch, \& Post, 1996), women showed more widespread activation of limbic areas during transient sadness than did men, despite comparable self-report ratings of mood. Of the two previous imaging studies investigating amygdala encoding of emotional experiences, one used female subjects and reported right-lateralized correlations (Cahill et al., 1996), whereas the other used male subjects and reported bilateral correlations (Hamann et al., 1999). In our own study, the study of female subjects revealed bilateral correlation patterns in the amygdala. Future studies should focus on gender differences in the encoding of emotional experiences, both within the amygdala and among a larger network of structures.

Another issue that will need to be resolved is how the experience of emotional valence or arousal modulates the encoding of emotional stimuli. Most previous imaging studies of the amygdala compared a negative test condition with a neutral control condition, in which case the negative condition differed from the neutral condition both in valence and in arousal. However, the experience of arousal can affect brain activation laterality in response to negative and positive valence (Canli et al., 1998), suggesting that both arousal and valence may play a role in encoding processes for emotional stimuli. Future work needs to address the conditions under which valence and arousal are critical dimensions affecting the encoding process and to define the neural systems mediating arousal influences on memory.

\section{REFERENCES}

Adolphs, R., Cahill, L., SChul, R., \& Babinsky, R. (1997). Impaired declarative memory for emotional material following bilateral damage in humans. Learning \& Memory, 4, 291-300.

Bechara, A., Tranel, D., Damasio, H., Adolphs, R., Rockland, C., \& Damasio, A. R. (1995). Double dissociation of conditioning and declarative knowledge relative to the amygdala and hippocampus in humans. Science, 269, 1115-1118.

Breiter, H. C., Etcoff, N. L., Whalen, P. J., Kennedy, W. A., Rauch, S. L., Buckner, R. L., Strauss, M. M., Hyman, S. E., \& Rosen, B. R. (1996). Response and habituation of the human amygdala during visual processing of facial expression. Neuron, 17, 875-887.

Cahill, L., Babinsky, R., Markowitsch, H. J., \& MCGaugh, J. L. (1995). The amygdala and emotional memory. Nature, 377, 295-296. Cahill, L., Haier, R. J., Fallon, J., Alkire, M. T., Tang, C., KeaTOR, D., Wu, J., \& MCGAUGH, J. L. (1996). Amygdala activity at encoding correlated with long-term, free recall of emotional information. Proceedings of the National Academy of Sciences, 93, 8016-8021.

Cahill, L., \& McGaugh, J. L. (1990). Amygdaloid complex lesions differentially affect retention of tasks using appetitive and aversive reinforcement. Behavioral Neuroscience, 104, 532-543.

Cahill, L., \& McGaugh, J. L. (1998). Mechanisms of emotional arousal and lasting declarative memory. Trends in Neurosciences, 21, 294-299.

CANLI, T., \& Brown, T. H. (1996). Amygdala stimulation enhances the rat eyeblink reflex through a short-latency mechanism. Behavioral Neuroscience, 110, 51-59.

Canli, T., Desmond, J. E., Zhao, Z., Glover, G., \& Gabrieli, J. D. E. (1998). Hemispheric asymmetry for emotional stimuli detected with fMRI. Neuroreport, 9, 3233-3239.

Davis, M. (1992). The role of the amygdala in conditioned fear. In J. P. Aggleton (Ed.), The amygdala: Neurobiological aspects of emotion, memory, and mental dysfunction (pp. 255-306). New York: Wiley-Liss.

Davis, M., Rainnie, D., \& Cassell, M. (1994). Neurotransmission in the rat amygdala related to fear and anxiety. TINS, 17, 207-214.

Desmond, J. E., \& Lim, K. O. (1997). On- and offline Talairach registration for structural and functional MRI studies. Human Brain Mapping, 5, 58-73.

Drevets, W. C., Videen, T. O., Price, J. L., Preskorn, S. H., CarMICHAEL, S. T., \& RAICHLE, M. E. (1992). A functional anatomical study of unipolar depression. Journal of Neuroscience, 12, 3628-3641.

Fink, G. R., Markowitsch, H. J., Reinkemeier, M., Bruckbauer, T., Kessler, J., \& Heiss, W.-D. (1996). Cerebral representation of one's own past: Neural networks involved in autobiographical memory. Journal of Neuroscience, 16, 4275-4282.

Fischer, H., Wik, G., \& Fredrikson, M. (1996). Functional neuroanatomy of robbery re-experience: Affective memories studied with PET. Neuroreport, 7, 2081-2086.

Fredrikson, M., Wik, G., Annas, P., Ericson, K., \& Stone-Elander, S. (1995). Functional neuroanatomy of visually elicited simple phobic fear: Additional data and theoretical analysis. Psychophysiology, 32, 43-48.

Fredrikson, M., Wik, G., Fischer, H., \& Andersson, J. (1995). Affective and attentive neural networks in humans: A PET study of Pavlovian conditioning. Neuroreport, 7, 97-101.

Fredrikson, M., WiK, G., Greitz, T., Eriksson, L., Stone-Elander, S., Ericson, K., \& Sedvall, G. (1993). Regional cerebral blood flow during experimental phobic fear. Psychophysiology, 30, 126-130. 
Friston, K. J., Jezzard, P., \& Turner, R. (1994). Analysis of functional MRI time-series. Human Brain Mapping, 1, 153-171.

George, M. S., Ketter, T. A., Parekh, P. I., Herscovitch, P., \& Post, R. M. (1996). Gender differences in regional cerebral blood flow during transient self-induced sadness or happiness. Biological Psychiatry, 40, 859-871.

Glover, G. H., \& SoNG, L. (1998). Self-navigated spiral fMRI: Interleaved versus single-shot. Magnetic Resonance in Medicine, 39, 361-368.

Gur, R. C., Mozley, L. H., Mozley, P. D., Resnick, S. M., KarP, J. S., Alavi, A., Arnold, S. E., \& GuR, R. E. (1995). Sex differences in regional cerebral glucose metabolism during a resting state. Science, 267, 528-531.

Hamann, S. B., Ely, T. D., Grafton, S. T., \& Kilts, C. D. (1999). Amygdala activity related to enhanced memory for pleasant and aversive stimuli. Nature Neuroscience, 2, 289-293.

Hugdahl, K., Berardi, A., Thompson, W. L., Kosslyn, S. M., Macy, R., Baker, D. P., Alpert, N. M., \& LeDouX, J. E. (1995). Brain mechanisms in human classical conditioning: A PET blood flow study. Neuroreport, 6, 1723-1728.

IKEgAYA, Y., SAITo, H., \& ABE, K. (1996). The basomedial and basolateral amygdaloid nuclei contribute to the induction of long-term potentiation in the dentate gyrus in vivo. European Journal of Neuroscience, 8, 1833-1839.

Irwin, W., Davidson, R. J., Lowe, M. J., Mock, B. J., Sorenson, J. A., \& TURSKI, P. A. (1996). Human amygdala activation detected with echo-planar functional magnetic resonance imaging. Neuroreport, 7 , 1765-1769.

Ketter, T. A., Andreason, P. J., George, M. S., Lee, C., Gill, D. S., Parekh, P. I., Willis, M. W., Herscovitch, P., \& Post, R. M. (1996). Anterior paralimbic mediation of procaine-induced emotional and psychosensory experiences. Archives of General Psychiatry, 53, 59-69.

LaBar, K. S., LeDoux, J. E., Spencer, D. D., \& Phelps, E. A. (1995). Impaired fear conditioning following unilateral temporal lobectomy in humans. Journal of Neuroscience, 15, 6846-6855.

Lane, R. D., Reiman, E. M., Ahern, G. L., Schwartz, G. E., \& DavidSON, R. J. (1997). Neuroanatomical correlates of happiness, sadness and disgust. American Journal of Psychiatry, 154, 926-933.

Lane, R. D., Reiman, E. M., Axelrod, B., Yun, L.-S., Holmes, A., \& SCHWARTZ, G. E. (1998). Neural correlates of levels of emotional awareness: Evidence of an interaction between emotions and attention in the anterior cingulate cortex. Journal of Cognitive Neuroscience, 10, $525-535$.

Lane, R. D., Reiman, E. M., Bradley, M. M., Lang, P. J., Ahern, G. L., Davidson, R. J., \& Schwartz, G. E. (1997). Neuroanatomical correlates of pleasant and unpleasant emotion. Neuropsychologia, 35, 1437-1444.

LANG, P. J., \& GreENwald, M. K. (1993). International affective picture system standardization procedure and results for affective judgments (Tech. Rep. 1A-1C). Jacksonville: University of North Florida, Center for Research in Psychophysiology.

Lang, P. J., Greenwald, M. K., Bradley, M. M., \& Hamm, A. O. (1993). Looking at pictures: Affective, facial, visceral, and behavioral reactions. Psychophysiology, 30, 261-273.

LeDoux, J. E. (1995). Emotion: Clues from the brain. Annual Review of Psychology, 46, 209-235.

McGaugh, J. L., Cahill, L., \& Roozendaal, B. (1996). Involvement of the amygdala in memory storage: Interaction with other brain systems. Proceedings of the National Academy of Sciences, 93, 1350813514.

Morris, J. S., Frith, C. D., Perrett, D. I., Rowland, D., Young, A.W., Calder, A. J., \& Dolan, R. J. (1996). A differential neural response in the human amygdala to fearful and happy facial expressions. Nature, 383, 812-815.

Muller, J., Corodimas, K. P., Fridel, Z., \& LeDoux, J. E. (1997) Functional inactivation of the lateral and basal nuclei of the amygdala by muscimol prevents fear conditioning to an explicit conditioned stimulus and to contextual stimuli. Behavioral Neuroscience, 111, 683-691.
Nagel, J. A., \& Kemble, E. D. (1976). Effects of amygdaloid lesions on the performance of rats in four passive avoidance tasks. Physiology \& Behavior, 17, 245-250.

Ogawa, S., Lee, T. M., Nayak, A. S., \& Glynn, P. (1990). Oxygenationsensitive contrast in magnetic resonance image of rodent brain at high magnetic fields. Magnetic Resonance Medicine, 14, 68-78.

Packard, M. G., Cahill, L., \& McGaugh, J. L. (1994). Amygdala modulation of hippocampal-dependent and caudate nucleus-dependent memory processes. Proceedings of the National Academy of Sciences, 91, 8477-8481.

PACKARD, M. G., \& McGaugh, J. L. (1996). Inactivation of hippocampus or caudate nucleus with lidocaine differentially affects expression of place and response learning. Neurobiology of Learning \& Memory, 65, 65-72.

Packard, M. G., \& Teather, L. A. (1998). Amygdala modulation of multiple memory systems: Hippocampus and caudate-putamen. Neurobiology of Learning \& Memory, 69, 163-203.

Pardo, J. V., Pardo, P. J., \& Raichle, M. E. (1993). Neural correlates of self-induced dysphoria. American Journal of Psychiatry, 150, 713719.

Phelps, E. A., \& ANDERson, A. K. (1997). Emotional memory: What does the amygdala do? Current Biology, 7, R311-R314.

Phillips, M. L., Young, A. W., Senior, C., Brammer, M., ANdrew, C., Calder, A. J., Bullmore, E. T., Perrett, D. I., Rowland, D., Williams, S. C. R., Gray, J. A., \& David, A. S. (1997). A specific neural substrate for perceiving facial expressions of disgust. Nature, 389, 495-498.

Rauch, S. L., Savage, C. R., Alpert, N. M., Miguel, E. C., Bear, L., Breiter, H. C., Fischman, A. J., Manzo, P. A., Moretti, C., \& JENIKE, M. A. (1995). A positron emission tomographic study of simple phobic symptom provocation. Archives of General Psychiatry, 52, 20-28.

Rauch, S. L., VAn Der Kolk, B. A., Fisler, R. E., Alpert, N. M., Orr, S. P., Savage, C. R., Fischman, A. J., Jenike, M. A., \& Pitman, R. K. (1996). A symptom provocation study of posttraumatic stress disorder using positron emission tomography and script-driven imagery. Archives of General Psychiatry, 53, 380-387.

Reiman, E. M., Lane, R. D., Ahern, G. L., Schwartz, G. E., DavidSon, R. J., Friston, K. J., YUn, L.-S., \& Chen, K. (1997). Neuroanatomical correlates of externally and internally generated human emotion. American Journal of Psychiatry, 154, 918-925.

Rogan, M. T., Staubli, U. V., \& LeDoux, J. E. (1997). Fear conditioning induces associative long-term potentiation in the amygdala. Nature, 390, 604-607.

Schneider, F., Grodd, W., Weiss, U., Klose, U., Mayer, K. R. NAGELE, T., \& GUR, R. C. (1997). Functional MRI reveals left amygdala activation during emotion. Psychiatry Research, 76, 75-82.

Schneider, F., Gur, R. E., Mozley, L. H., Smith, R. J., Mozley, P. D. Censits, D. M., Alavi, A., \& Gur, R. C. (1995). Mood effects on limbic blood flow correlate with emotional self-rating: a PET study with oxygen-15 labeled water. Psychiatry Research: Neuroimaging, 61, 265-283.

SHIELDS, S. A. (1991). Gender in the psychology of emotion: A selective research review. In K. T. Strongman (Ed.), International review of studies on emotion (pp. 227-245). New York: Wiley.

TALAIRACH, J., \& TOURNOUX, P. (1988). Co-planar stereotaxic atlas of the human brain. New York: Thieme Medical.

Taylor, S. F., Liberzon, I., Fig, L. M., Decker, L. R., Minoshima, S., \& KOEPPE, R. A. (1998). The effect of emotional content on visual recognition memory: A PET activation study. Neuroimage, 8, 188-197.

Weisz, D. J., HaRDEN, D. G., \& XiANG, Z. (1992). Effects of amygdala lesions on reflex facilitation and conditioned response acquisition during nictitating membrane response conditioning in rabbit. Behavioral Neuroscience, 106, 262-273.

Whalen, P. J., \& KaPP, B. S. (1991). Contributions of the amygdaloid central nucleus to the modulation of the nictitating membrane reflex in the rabbit. Behavioral Neuroscience, 105, 141-153.

Wik, G., Fredrikson, M., Ericson, K., Eriksson, L., StoneElander, S., \& Greitz, T. (1993). A functional cerebral response to 
frightening visual stimulation. Psychiatry Research: Neuroimaging, 50, 15-24.

Woods, R. P., Cherry, S. R., \& Mazziotta, J. C. (1992). Rapid automated algorithm for aligning and reslicing PET images. Journal of Computer Assisted Tomography, 16, 620-633.

Xiong, J., GaO, J.-H., LANCASTER, J. L., \& Fox, P. T. (1995). Clustered pixel analysis for functional MRI activation studies of the human brain. Human Brain Mapping, 3, 287-301.

\section{NOTE}

1. Another interpretation is that the anterior cingulate inhibits the other structures (the amygdala, the insula, and the middle frontal gyrus), so that greater reactivity in the cingulate during exposure to positive pictures would lead to reduced reactivity in the other three structures to positive pictures. Given that the literature offers more evidence for an increase in amygdala activation to negative stimuli than for a decrease in amygdala activation to positive stimuli, we shall focus on the consequences of greater amygdala activation to negative stimuli.

\section{APPENDIX \\ Lists (in Ascending Order) of IAPS Pictures Used}

During encoding: $108,109,127,128,151,161,171,204$ $205,211,212,215,216,226,263,270,271,280,303,316,322$, $461,465,661,680,694,720,723,733,757,803,804,809,813$, $822,823,900,914,916,944$.

During the recognition test: $107,108,109,111,112,120$, $122,127,128,151,161,162,171,174,204,205,207,211,212$, $215,216,217,225,226,237,251,263,270,271,273,280,303$, $315,316,322,323,461,465,500,501,522,560,575,582,583$, $602,619,621,641,661,680,694,720,723,726,727,728,733$, $735,736,757,758,803,804,809,813,822,823,851,900,914$, $916,918,919,929,940,942,943,944,950$.

(Manuscript received March 16, 1999; revision accepted for publication June 24,1999 .) 